\title{
Magnetorotational supernovae with different equations of state
}

\section{Sergey G. Moiseenko and Gennady S. Bisnovatyi-Kogan}

\author{
Space Research Institute, \\ Profsouznaya str. 84/32 117997 Moscow, Russia \\ email: moiseenko@iki.rssi.ru
}

\begin{abstract}
We present results of the simulation of a magneto-rotational supernova explosion. We show that, due to the differential rotation of the collapsing iron core, the magnetic field increases with time. The magnetic field transfers angular momentum and a MHD shock wave forms. This shock wave produces the supernova explosion. The explosion energy computed in our simulations is $0.5-2.5 \cdot 10^{51} \mathrm{erg}$. We used two different equations of state for the simulations. The results are rather similar.
\end{abstract}

Keywords. supernovae: general, methods: numerical

The important role of the rotation and the magnetic fields for core collapse supernova was suggested in the paper by Bisnovatyi-Kogan (1970). The idea of the magnetorotational (MR) mechanism consists of angular momentum transfer outwards using the magnetic field, which is twisting due to the differential rotation. The toroidal component of the magnetic field is amplifying with time, which leads to increasing of the magnetic pressure and generating the supernova shock.

According to the results of two-dimensional modeling for the MR supernova mechanism, the initial stage of the linear growth of the toroidal magnetic field is followed by an exponential increase in both the toroidal and poloidal field components, accompanied by the development of MR instability.

For the simulations we used a specially developed implicit Lagrangian numerical scheme on a triangular grid of variable structure Ardeljan et al. (1996).

The results of our simulations show that the explosion energy depends weakly on the strength of the initial magnetic field. The 'shape' of the explosion depends qualitatively on the configuration of the magnetic field. For the quadrupole-like field the explosion develops preferably near the equatorial plane Ardeljan et al. (2005). For the dipolelike initial magnetic field the MR supernova explosion develops a mildly collimated jet Moiseenko et al. (2006).

In the paper by Bisnovatyi-Kogan et al. (2008) we simulated a MR supernova explosion for various initial core masses and rotational energies. The initial core mass was varied from $1.2 M_{\odot}$ to $1.7 M_{\odot}$. The specific rotational energy at the time when the magnetic field was switched on, $E_{\text {rot }} / M_{\text {core }}$, was varied from $0.19 \times 10^{19}$ to $0.4 \times 10^{19} \mathrm{erg} / \mathrm{g}$. Fig. 1 presents the results of our calculations. It shows that the explosive energy of a MR supernova increases substantially with the mass of the iron core and the initial rotational energy (angular velocity; see Table). It is obvious that the energy released in a MR supernova is sufficient to explain supernovae with collapsing cores: $(0.5-2.6) 10^{51} \mathrm{erg}$ (Type II and Ib supernovae). The energies of Type Ic supernovae can be higher, probably due to the collapse of more massive cores, of the order of several tens of $M_{\odot}$.

For the simulations we used two different equations of state. The first one (Ardelyan et al. (1987)) is the approximation of tables from Ivanova et al. (1969). The second one 


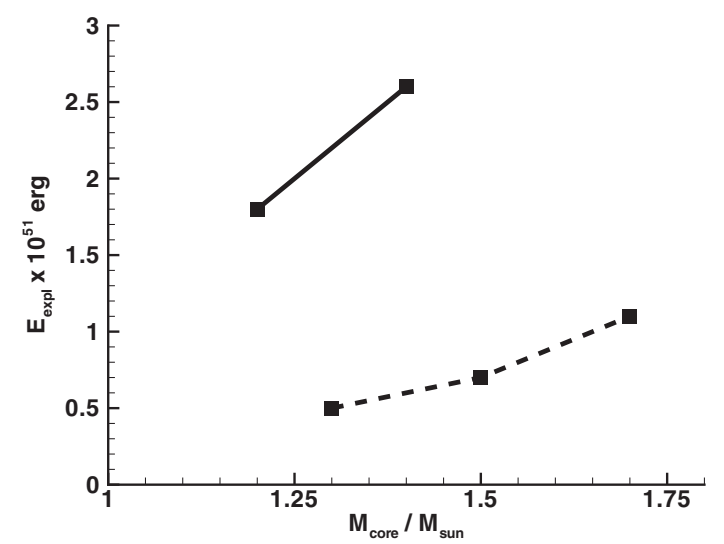

Figure 1. Energy of the explosion of a MR supernova as a function of the initial mass of the core for various specific rotational energies before the start of the evolution of the magnetic field (before beginning of the magnetic field evolution), $E_{\text {rot }} / M_{\text {core }} \approx 0.39-0.40 \cdot 10^{19} \mathrm{erg} / \mathrm{g}$ (solid line) and $E_{\text {rot }} / M_{\text {core }} \approx 0.19-0.23 \cdot 10^{19} \mathrm{erg} / \mathrm{g}$ (dashed line) (before collapse).

is from the paper by Shen et al. (1998). For the application of the second EOS we follow the paper by Kotake et al. (2003).

The results of simulations of the MR supernova explosion for both equations of state are in good agreement. The details of the simulations of the MR supernova with the EOS by Shen et al. (1998) will be published elsewhere.

\section{Acknowledgements}

This work was partially supported by RFBR grant 11-02-00602, grant NsH-5440.2012.2, program of the RAS 'Origin, structure and evolution of objects in the Universe'.

\section{References}

Ardeljan, N. V., Bisnovatyi-Kogan, G. S., Kosmachevskii, K. V., \& Moiseenko, S. G., 1996, $A \& A S, 115,573$

Ardelyan, N. V., Bisnovatyi-Kogan, G. S., Popov, Yu. P., \& Chernigovsky, S. V., 1987, Astron. Zh. 64, 761 (Soviet Astronomy, 1987, 31, 398)

Ardeljan, N. V., Bisnovatyi-Kogan, G. S., \& Moiseenko, S. G., Monthly Not. Roy. Astron. Soc., 359, 333 (2005).

Bisnovatyi-Kogan, G. S., 1970, Astron. Zh. 47, 813 (Soviet Astronomy, 1971, 14, 652

Bisnovatyi-Kogan G. S., Moiseenko S. G., \& Ardelyan N. V., 2008, Astronomy Reports, 52,997

Ivanova, L. N., Imschennik, V. S., \& Nadyozhin D. K., 1969 Nauch. inf. Astron. Soveta, 13,3

Kotake, K., Yamada, S., \& Sato, K. 2003, ApJ, 595, 304

Moiseenko S. G., Bisnovatyi-Kogan G. S., \& Ardeljan N. V., 2006, Monthly Not. Roy. Astron. Soc., 370,501

Shen, H., Toki, H., Oyamatsu, K., \& Sumiyoshi, K. 1998, Nucl. Phys. A, 637, 435 